\title{
Circulating Cell-free miRNA Expression and its Association with Clinicopathologic Features in Inflammatory and Non- Inflammatory Breast Cancer
}

\author{
K Hamdi $^{1 *}$, J Blancato ${ }^{2}$, D Goerlitz ${ }^{2}$, MD Islam ${ }^{2}$, B Neili ${ }^{1}$, A Abidi ${ }^{1}$, A Gat ${ }^{1}$, F Ben \\ Ayed $^{3}$, S Chivi ${ }^{2}$, CA Loffredo ${ }^{2}$, I Jillson ${ }^{2}$, A Benammar Elgaaied ${ }^{1}$, R Marrakchi $^{1}$
}

\begin{abstract}
Recent discovery showing the presence of microRNAs (miRNAs) in the circulation sparked interest in their use as potential biomarkers. Our previous studies showed the diagnostic potential of miR-451 as a serological marker for inflammatory breast cancer (IBC), miR-337- 5p and miR-30b for non-inflammatory breast cancer (non-IBC). The aim of this study is to investigate the prognostic values of circulating miRNAs by comparing the amounts of 12 circulating miRNAs in the serum of IBC and non-IBC from Tunisian breast cancer patients, and by determinating whether correlated pairs of miRNAs could provide useful information in the diagnosis of IBC and non-IBC patients. TaqMan qPCR was performed to detect circulating expression of miRNAs in serum of 20 IBC, 20 non-IBC and 20 healthy controls. Nonparametric rank Spearman rho correlation coefficient was used to examine the prognostic value of miRNAs and to assess the correlation profile between miRNAs expression. Further, a large number of miRNAs were highly correlated (rho $>0.5)$ in both patients groups and controls. Also, the correlations profiles were different between IBC, non-IBC and healthy controls indicating important changes in molecular pathways in cancer cells. Our results showed that miR-335 was significantly overexpressed in premenopausal non-IBC patients; miR-24 was significantly overexpressed in non-IBC postmenopausal patients. Patients with previous parity had higher serum of miR-342-5p levels than those without. Furthermore, patients with HER2+ IBC present lower serum levels of miR-15a than patients with HER2- disease. Together, these results underline the potential of miRNAs to function as diagnostic and prognostic markers for IBC and non-IBC, with links to the menopausal state, Her2 status and parity.
\end{abstract}

Keywords: Breast cancer - inflammatory breast cancer - circulating miRNAs - biomarker. prognostic - diagnostic

Asian Pac J Cancer Prev, 17 (4), 1801-1810

\section{Introduction}

MicroRNAs (miRNAs) are small noncoding RNA oligonucleotides (22-25 nucleotides) that suppress gene expression post-transcriptionally via sequence-specific interactions with cognate mRNA targets, they suppress translation and induce degradation of mRNAs. Because of their broad targeting range, miRNAs are expected to be involved in most cellular processes. Therefore, dysregulation of the expression of miRNAs results in diverse disease states (Sayed and Abdellatif, 2011). Aberrations in miRNAs expression levels influence the occurrence and development of tumor and have been linked to the onset and progression of various types of cancer (Palmero et al., 2011). In this particular context, the roles of miRNAs in carcinogenesis and tumor progression are mainly associated with miRNA-regulated expression of oncogenes and tumor suppressor genes (Croce, 2009). In fact, the overexpression of certain miRNAs could lead to the repression of tumor suppressor genes. Willingly, miRNAs down-regulation could result in increased oncogene expression ( $\mathrm{Li}$ et al., 2013). Importantly, in previous work it has been demonstrated that extracellular miRNAs levels are surprisingly stable in body fluids ,retrievable and measurable from fresh or archival serum and plasma samples (Chen et al., 2008; Mitchell et al., 2008; Schwarzenbach et al., 2014).

Since then, accumulated studies have convincingly demonstrated that the extracellular miRNAs are released into the blood circulation by apoptotic and necrotic cells (Schwarzenbach et al., 2011) or high density lipoproteine ( HDL) particles, and exist either cell-freely, complexed with AGO proteins, or in exosomes in the blood circulation (Simpson et al., 2009). In the case of cancer disease, 


\section{K Hamdi et al}

the use of a less invasive method than tissue biopsy is required and it may represent a major difference in clinical outcome. Hence, a large number of studies have assessed the potential use of circulating miRNAs as biomarkers in various types of cancer, including breast cancer, gastric, hepatocellular, non-small cell lung cancer (Konishi et al., 2012; Tomimaru et al., 2012; Hagrass et al., 2015), Table 1.

Breast cancer is a malignant breast neoplasm originating from breast tissues, and it's the cause of cancer related mortality among women worldwide. Breast cancer is a highly heterogeneous disease that has multiple subtypes with distinct clinical outcomes. Inflammatory breast carcinoma (IBC) is the most aggressive manifestation of primary breast carcinoma (Yamauchi et al., 2012). Simultaneously, IBC patients have worse survival outcomes compared with noninflammatory breast cancer patients even with multiple improved treatments, such as polychemotherapy and endocrine regimens (Dawood and Cristofanilli, 2011; Dawood et al., 2011). Our previous miRNAs expression analysis revealed that miR-451, miR-342-3p, miR$30 \mathrm{~b}$, miR-24 and miR-337-5p were dysregulated in inflammatory and non-inflammatory mammary tumors when compared to healthy controls as well as among the two groups of breast cancer. Serum levels of miR-451 were significantly downregulated in IBC compared with healthy controls. While serum levels of miR-337-5p and miR-30 were significantly over-expressed in non-IBC samples compared with healthy controls, suggests that these miRNAs may be used as a biomarker for diagnosis of IBC and non-IBC (Hamdi et al., 2014). These results promoted us to investigate the possible role of these miRNAs in breast cancer carcinogenesis.

Heneghan and co-investigators demonstrated that circulating miR-195 was found to be breast cancer specific and it could significantly differentiate breast cancer from other cancers and healthy controls. Furthermore, serum levels of miR-195 were correlated with nodal and estrogen-receptor status (Heneghan et al., 2010) .In addition, miR-155 may be differentially expressed in the serum of women with hormone sensitive compared to women with hormone insensitive breast cancer (Zhu et al., 2009).

Interestingly, another study of breast cancer demonstrated that patients at advanced tumor stages had significantly higher miR-34a than patients at early tumor stages. In the metastatic patients, miR-10b, miR-34a, and miR-155 correlated with the presence of overt metastases circulating microRNAs (Roth et al., 2010).

In the current study, we aimed to investigate the association of 12 serum miRNAs expression levels with clinicopathologic features in IBC and non-IBC serum, and by determining whether correlated pairs of miRNAs could provide useful information in the discrimination between IBC and non-IBC. The findings may increase our understanding of the miRNAs signature in IBC and non-IBC and suggest a potential diagnostic or prognostic strategy for targeting specific miRNAs.

\section{Materials and Methods}

\section{Blood samples}

Experiments were conducted in accordance with the principles of Declaration of Helsinki. This study was approved by the Medical Ethics Committee of Pasteur Institute of Tunis and the Georgetown University Institutional Review Board. Written informed consents were obtained from all the participants.

Serum samples were collected from 60 women diagnosed and treated at Hannibal International Clinic of Tunis from March 2011 and January 2012 before they underwent any therapeutic procedure : 20 with non-IBC, 20 with IBC, and 20 cancer-free women were enrolled in our study. Control blood samples were collected at the same period from healthy women volunteers with no history of malignant diseases, no blood donations received in the previous 3 years, and no current inflammatory conditions.

$5 \mathrm{ml}$ of blood was collected from each woman directly into serum collection tubes. The whole blood was allowed to stand for about $1 \mathrm{~h}$ at RT before being centrifuged at $1200 \mathrm{~g}$ for 10 minutes at $4^{\circ} \mathrm{C}$ followed by careful separation of serum. Another centrifugation was performed for $10 \mathrm{~min}$ at $10,000 \times \mathrm{g}$ at $4^{\circ} \mathrm{C}$ to remove the cellular debits. The resultant serum was aliquoted into eppendorf tubes and stored at $-80^{\circ} \mathrm{C}$.

TNM stage was assessed according to the 7 th edition of the American Joint Committee on Cancer (AJCC) staging manual. Samples were stained histologically to determine

\section{Table 2. Tumour Characteristics}

\begin{tabular}{|c|c|c|c|}
\hline $\begin{array}{l}\text { Clinicopathological } \\
\text { features }\end{array}$ & $\begin{array}{c}\text { IBC } \\
(\mathrm{N}=20)\end{array}$ & $\begin{array}{l}\text { non-IBC } \\
(\mathrm{N}=20)\end{array}$ & P-value \\
\hline \multicolumn{4}{|l|}{ Patients' ages (years) } \\
\hline Mean & 49 & 50.2 & \\
\hline Range & $34-70$ & $29-78$ & 0.236 \\
\hline \multicolumn{4}{|l|}{ Tumour stage } \\
\hline I & $0(0 \%)$ & $0(0 \%)$ & \\
\hline II & $0(0 \%)$ & $6(30 \%)$ & \\
\hline III & $0(0 \%)$ & $13(65 \%)$ & \\
\hline IV & IVd20(100\%) & $\operatorname{IVb} 1(5 \%)$ & \\
\hline \multicolumn{4}{|c|}{ Histological tumour gradea } \\
\hline I & $0(0 \%)$ & $0(0 \%)$ & \\
\hline II & $9(45 \%)$ & $7(35 \%)$ & 0.264 \\
\hline III & $11(55 \%)$ & $13(65 \%)$ & \\
\hline \multicolumn{4}{|l|}{ Node status } \\
\hline No & $2(10 \%)$ & $7(35 \%)$ & \\
\hline N1 & $16(80 \%)$ & $13(65 \%)$ & 0.064 \\
\hline N2 & $2(10 \%)$ & 0 & \\
\hline N3 & $0(0 \%)$ & 0 & \\
\hline \multicolumn{4}{|l|}{ Distant metastasis } \\
\hline M0 & $14(70 \%)$ & $4(20 \%)$ & \\
\hline M1 & $0(0 \%)$ & $2(10 \%)$ & 0.001 \\
\hline Unknown & $6(30 \%)$ & $14(70 \%)$ & \\
\hline \multicolumn{4}{|l|}{ Oestrogen receptor } \\
\hline Positive & $3(15 \%)$ & $11(55 \%)$ & 0.0018 \\
\hline Negative & $17(85 \%)$ & $9(35 \%)$ & \\
\hline \multicolumn{4}{|l|}{ HER2 amplification } \\
\hline Positive & $13(65 \%)$ & $13(65 \%)$ & 0.2 \\
\hline
\end{tabular}


histological grade, which was classified into low, moderate and poor grade according to criteria modified by Elston and Ellis. The status of ER, PR and HER2 were detected by immunohistochemistry (IHC). Tumor characteristics are provided in Table 2 .

\section{RNA extraction}

Total RNA was extracted from $500 \mu$ serum using mirVana miRNA Isolation Kit (Life Technologies) according to the manufacturer's instructions. The quality and yield of each RNA sample was measured using a NanoDrop ND-1000 Spectrophotometer. The RNA specimens were stored at $-80^{\circ} \mathrm{C}$ until reverse transcription.

\section{Reverse Transcription and Real-Time PCR}

In the reverse transcription (RT) step, cDNA is reverse transcribed from total RNA samples using specific miRNA primers from the TaqMan MicroRNAAssays and reagents from the TaqMan ${ }^{\circledR}$ MicroRNA Reverse Transcription Kit (Applied Biosystems, Life Technologies). The amount of RNAs used for RT was $2 \mathrm{ng}$ and the cDNA specimens were stored at $-20^{\circ} \mathrm{C}$ until PCR. In the PCR step, PCR products are amplified from cDNA samples using the TaqMan MicroRNA Assay together with the TaqMan ${ }^{\circledR}$ Universal
PCR Master Mix . The PCR reactions were performed on 7900 Real-Time PCR system following the manufacturer's protocol. (Applied Biosystems, United States). For each sample, the miRNAs expression levels were assayed in triplicate and were run with endogenous control RNU-48 (Control miRNAs Assay, Applied Biosystems, USA). also in triplicate, with a single no template negative control reaction. The normalization step was performed according to the 2-((Mean $\Delta \mathrm{Ct}$ Target)-(Mean $\Delta \mathrm{Ct}$ Calibrator)) method (Livak and Schmittgen, 2001).

Statistical analysis was performed using SPSS 20.0 statistical software package for Windows.

Mann-Whitney U nonparametric test was used for comparing two different groups such as premenopausal and postmenopausal. Coefficient correlations were calculated using Spearman's rho test to find a correlation between two variables.. All P values lower than 0.05 were considered statistically significant.

\section{Bioinformatics Tools for Predicting miRNA Targets}

The predictive potential target genes of miRNAs were searched in 6 on-line databases, including MIRBD, HSA-MIRBase, TARGETSCAN,TARGETMINER,

Table 1. A compendium of Circulating miRNAs with Potential as Biomarkers for Breast Cancer and other Human Malignancies

\begin{tabular}{|c|c|c|c|c|}
\hline miRNAs & $\begin{array}{l}\text { Cancer } \\
\text { type }\end{array}$ & Body fluid source & Clinical correlations & Reference \\
\hline $\operatorname{miR}-335$ & Breast & Serum & $\begin{array}{l}\text { Overexpressed miR-335 were associated with } \\
\text { breast cancer } \\
\text { histological tumor grades and hormone receptor } \\
\text { expression status }\end{array}$ & (Wang et al., 2010) \\
\hline \multirow{3}{*}{$\operatorname{miR}-24$} & Ovarian & Plasma & lower levels of miR-24 in patients than controls & \multirow{3}{*}{$\begin{array}{c}\text { (Wu et al., 2012; Shapira } \\
\text { et al., 2014; Fang et al., } \\
\text { 2015) }\end{array}$} \\
\hline & Breast & Serum & $\begin{array}{c}\text { Increased serum level of miR-24 in Breast } \\
\text { Cancer patients. }\end{array}$ & \\
\hline & Colorectal & Plasma & $\begin{array}{l}\text { Decreased plasma levels of miR-24 serve as } \\
\text { novel biomarkers for early stage detection of } \\
\text { colorectal }\end{array}$ & \\
\hline $\operatorname{miR}-320$ & Breast & Serum & $\begin{array}{l}\text { Underexpressed in the serum of women who } \\
\text { developed lymph node-positive breast tumors. }\end{array}$ & (Yan et al., 2008) \\
\hline miR-548d-5p & Bladder & Urine & $\begin{array}{c}\text { Expressed predominantly in patients with higher } \\
\text { cancer stages }\end{array}$ & (Brisuda et al.,2014) \\
\hline $\operatorname{miR}-342-5 p$ & Ovarian & Whole blood & Decreased levels in ovarian cancer & (Hausler et al., 2010) \\
\hline miR-15a & Breast & Serum & $\begin{array}{l}\text { Increased serum level in older compared to } \\
\text { young breast patients }\end{array}$ & (Kodahl et al., 2014) \\
\hline $\operatorname{miR}-342-3 p$ & Breast & Serum & $\begin{array}{l}\text { High serum level in older compared to young } \\
\text { breast patients }\end{array}$ & (Hatse et al., 2014) \\
\hline miR-29a & Colorectal & Plasma & $\begin{array}{c}\text { Increased plasma levels in advanced-stage can- } \\
\text { cer than controls }\end{array}$ & (Huang et al., 2010) \\
\hline $\operatorname{miR}-451$ & Breast & Plasma & $\begin{array}{l}\text { Increased plasma level found in breast cancer } \\
\text { patients }\end{array}$ & $\begin{array}{l}\text { (Van der Auwera et al., } \\
\text { 2010) }\end{array}$ \\
\hline $\operatorname{miR}-30 b$ & Breast & Serum & $\begin{array}{l}\text { high levels in the serum of breast cancer patients } \\
\text { than control subjects }\end{array}$ & (Hamdi et al.,2014) \\
\hline miR-337-5p & ESCC & Serum & $\begin{array}{l}\text { Serum levels were differentially expressed be- } \\
\text { tween ESCC patients and controls }\end{array}$ & (Wu et al., 2014) \\
\hline \multirow{2}{*}{$\operatorname{miR}-486-3 p$} & Prostate & Serum & $\begin{array}{l}\text { Serum level were significantly higher in patients } \\
\text { compared to controls }\end{array}$ & \multirow{2}{*}{$\begin{array}{l}\text { (Lodes et al., 2009; } \\
\text { Cortez et al., 2011; Hatse } \\
\text { et al., 2014) }\end{array}$} \\
\hline & Breast & Serum & $\begin{array}{c}\text { Increased serum level in older versus young } \\
\text { breast cancer patients }\end{array}$ & \\
\hline
\end{tabular}


TARGETSCAN-VERT and KEGG PATHWAY Database.

\section{Results}

Correlations between Expression of Different miRNAs.

The expression levels of twelve different miRNAs that were known to be deregulated in various cancers were quantified by real-time PCR analysis. In total, 20 serum samples from non-inflammatory, 20 from inflammatory breast cancer cases and 20 healthy controls were included in the analysis. Patients were recruited from Hannibal International Clinic of Cancerology in Tunis. In the first step, they were classified on the basis of their clinical and histopathological profiles, according to the tumor size, axillary's lymph node status, and histological grade, ER expression and human epidermal growth factor receptor 2 (HER2) amplification. There were no differences between the groups, the only difference between the groups was related to Oestrogen receptor status $(\mathrm{p}=0.0018)$ and metastasis $(\mathrm{p}=0.001)$. Tumor characteristics are provided in Table 2.

In complementary to serum miRNAs expression analysis in IBC and non-IBC groups, we assessed not only individual changes in mean miRNAs expression levels among groups, but changes in how miRNAs were correlated with each other across groups. For this analysis, non parametric rank Spearman rho correlation showed that a large number of miRNAs were highly (rho $>0.5$ ) correlated in both patients groups and controls.

In non-inflammatory breast cancer group, all miRNAs analyzed in the current study were highly correlated with each other. Interestingly, absolute correlation was scouted between miR-15a and miR-486-3p (Spearman's rho 1.000; $\mathrm{p}=0.000)$. Likewise, miR-320 was highly correlated with miR-24 and miR-548d-5p (Spearman's rho, 0.839 and 0.823 respectively; $\mathrm{p}=0.000)$. Moreover, expression of miR-451 were correlated with miR-24 (Spearman's

Table 3. Spearman Rank Correlation Coefficients between miRNA in IBC and non-IBC Samples

\begin{tabular}{|c|c|c|c|c|c|c|c|c|c|c|c|c|}
\hline \multicolumn{13}{|c|}{ non-IBC } \\
\hline & $\begin{array}{c}\text { miR- } \\
335\end{array}$ & $\begin{array}{c}\text { miR- } \\
24\end{array}$ & $\begin{array}{c}\text { miR- } \\
320\end{array}$ & $\begin{array}{c}\text { miR- } \\
548 d- \\
5 p\end{array}$ & $\begin{array}{l}\text { miR- } \\
342-5 p\end{array}$ & $\begin{array}{c}\mathrm{miR}- \\
15 \mathrm{a}\end{array}$ & $\begin{array}{c}\text { miR- } \\
342-3 p\end{array}$ & $\begin{array}{c}\mathrm{miR}- \\
29 \mathrm{a}\end{array}$ & $\begin{array}{c}\text { miR- } \\
451\end{array}$ & $\begin{array}{l}\mathrm{miR}- \\
30 \mathrm{~b}\end{array}$ & $\begin{array}{l}\text { miR- } \\
337-5 p\end{array}$ & $\begin{array}{c}\mathrm{miR} 486- \\
3 \mathrm{p}\end{array}$ \\
\hline miR-335 & & -0.16 & 0.3 & 0.52 & -0.23 & -0.11 & 0.15 & 0.23 & 0.04 & 0.21 & 0.34 & -0.11 \\
\hline miR-24 & 0.48 & & $0,83 * *$ & 0.59 & 0.35 & 0.59 & 0.55 & 0.49 & $0,61 *$ & 0.43 & 0.23 & 0.59 \\
\hline $\operatorname{miR}-320$ & 0.69 & 0.36 & & $0,82 * *$ & 0.3 & $0.53 *$ & 0.65 & 0.74 & 0.68 & 0.44 & 0.39 & 0.44 \\
\hline $\operatorname{miR} 548 d-5 p$ & 0.16 & 0.09 & 0.45 & & 0.1 & 0.3 & 0.66 & 0.62 & 0.49 & 0.53 & 0.54 & 0.3 \\
\hline $\operatorname{miR}-342-5 p$ & 0.07 & 0.39 & 0.06 & -0.23 & & 0.55 & $0.72 * *$ & 0.43 & 0.26 & 0.35 & 0.1 & 0.55 \\
\hline miR-15a & 0.62 & 0.59 & $0,59 *$ & 0.06 & 0.55 & & 0.27 & 0.43 & 0.19 & 0.35 & 0.36 & $1 * *$ \\
\hline $\operatorname{miR}-342-3 p$ & 0.36 & 0.08 & 0.25 & -0.1 & 0.57 & 0.57 & & 0.6 & 0.59 & 0.36 & 0.37 & 0.27 \\
\hline miR-29a & 0.34 & 0.28 & 0.21 & -0.08 & 0.33 & 0.59 & 0.57 & & 0.69 & 0.58 & 0.46 & 0.43 \\
\hline $\operatorname{miR}-451$ & 0.57 & 0.76 & 0.27 & -0.26 & 0.34 & 0.69 & 0.32 & 0.63 & & 0.51 & 0.52 & 0.19 \\
\hline miR-30b & 0.48 & 0.08 & 0.34 & -0.23 & -0.1 & 0.29 & 0.22 & 0.21 & 0.2 & & $0,58 *$ & 0.35 \\
\hline miR-337-5p & 0.49 & 0.5 & 0.12 & -0.22 & 0.56 & 0.39 & 0.49 & 0.06 & 0.47 & 0.15 & & 0.36 \\
\hline miR-486-3p & 0.07 & 0.39 & -0.03 & -0.23 & $1 * *$ & 0.55 & 0.57 & 0.33 & 0.34 & -0.1 & 0.6 & \\
\hline \multicolumn{13}{|c|}{ IBC } \\
\hline
\end{tabular}

*Correlation coefficients with indicate significant at the 0.05 level; ** Correlation coefficients with indicate significant at the 0.01 level

Table 4. Spearman Rank Correlation Coefficients between miRNA in healthy controls

\begin{tabular}{|c|c|c|c|c|c|c|c|c|c|c|c|c|}
\hline \multicolumn{13}{|c|}{ Healty Controls } \\
\hline & $\begin{array}{c}\text { miR- } \\
335 \\
\end{array}$ & $\begin{array}{c}\text { miR- } \\
24 \\
\end{array}$ & $\begin{array}{c}\text { miR- } \\
320 \\
\end{array}$ & $\begin{array}{c}\text { miR- } \\
548 d-5 p\end{array}$ & $\begin{array}{c}\text { miR- } \\
342-5 p\end{array}$ & $\begin{array}{c}\text { miR- } \\
15 \mathrm{a} \\
\end{array}$ & $\begin{array}{c}\text { miR- } \\
342-3 p\end{array}$ & $\begin{array}{c}\text { miR- } \\
29 a \\
\end{array}$ & $\begin{array}{c}\text { miR- } \\
451 \\
\end{array}$ & $\begin{array}{c}\text { miR- } \\
30 \mathrm{~b}\end{array}$ & $\begin{array}{c}\text { miR- } \\
337-5 p\end{array}$ & $\begin{array}{c}\operatorname{miR} 486- \\
3 p\end{array}$ \\
\hline miR-335 & - & 0.41 & 0.37 & 0.15 & 0.47 & -0.26 & 0.25 & 0.13 & 0.41 & 0.18 & 0.28 & 0.25 \\
\hline miR-24 & - & - & 0.11 & 0.18 & 0.39 & 0.56 & 0 & -0.28 & -0.07 & 0.45 & 0.2 & -0.06 \\
\hline $\operatorname{miR}-320$ & - & - & - & 0.65 & 0.61 & 0.21 & 0.25 & 0.12 & 0.54 & 0.5 & 0.31 & 0.25 \\
\hline miR548d-5p & - & - & - & - & 0.63 & 0.25 & -0.04 & 0.1 & 0.66 & 0.39 & 0.51 & -0.41 \\
\hline $\operatorname{miR}-342-5 p$ & - & - & - & - & - & -0.24 & $0,82 * *$ & 0.32 & 0.31 & 0.56 & 0.48 & 0.2 \\
\hline $\operatorname{miR}-15 a$ & - & - & - & - & - & - & -0.09 & 0.25 & -0.1 & -0.31 & -0.06 & -0.09 \\
\hline $\operatorname{miR}-342-3 p$ & - & - & - & - & - & - & - & 0.3 & -0.18 & 0.18 & 0.31 & $1 * *$ \\
\hline miR-29a & - & - & - & - & - & - & - & - & 0.13 & 0.14 & 0.16 & 0.3 \\
\hline $\operatorname{miR}-451$ & - & - & - & - & - & - & - & - & - & 0.12 & 0.52 & 0.18 \\
\hline miR-30b & - & - & - & - & - & - & - & - & - & - & 0.49 & 0.18 \\
\hline miR-337-5p & - & - & - & - & - & - & - & - & - & - & - & 0.15 \\
\hline $\operatorname{miR}-486-3 p$ & - & - & - & - & - & - & - & - & - & - & - & - \\
\hline
\end{tabular}

*Correlation coefficients with indicate significant at the 0.05 level; ** Correlation coefficients with indicate significant at the 0.01 level 
Table 5. Relationship between miRs expression and clinical and histopathology presentations in non-inflammatory breast cancer patients.

\begin{tabular}{|c|c|c|c|c|c|c|c|c|c|c|c|c|c|}
\hline $\begin{array}{l}\text { Patients } \\
\text { presentations }\end{array}$ & $\mathrm{N} 0$ & $\begin{array}{l}\mathrm{miR}- \\
335\end{array}$ & $\begin{array}{c}\mathrm{miR}- \\
24\end{array}$ & $\begin{array}{l}\mathrm{miR}- \\
320\end{array}$ & $\begin{array}{c}\text { miR- } \\
548 \mathrm{~d}- \\
5 \mathrm{p}\end{array}$ & $\begin{array}{c}\text { miR- } \\
342- \\
5 \mathrm{p} \\
\end{array}$ & $\begin{array}{c}\mathrm{miR}- \\
15 \mathrm{a}\end{array}$ & $\begin{array}{c}\text { miR- } \\
342- \\
3 p\end{array}$ & $\begin{array}{c}\mathrm{miR}- \\
29 \mathrm{a}\end{array}$ & $\begin{array}{l}\mathrm{miR}- \\
451\end{array}$ & $\begin{array}{l}\mathrm{miR}- \\
30 \mathrm{~b}\end{array}$ & $\begin{array}{c}\text { miR- } \\
337- \\
5 \mathrm{p}\end{array}$ & $\begin{array}{c}\mathrm{miR}- \\
486- \\
3 \mathrm{p} \\
\end{array}$ \\
\hline \multicolumn{14}{|l|}{ Age } \\
\hline$\geq 40$ years & 7 & 0.598 & 0.257 & 0.064 & 0.548 & 0.072 & 0.442 & 0.187 & 0.249 & 0.193 & 0.094 & 0.297 & 0.774 \\
\hline$<40$ years & 13 & & & & & & & & & & & & \\
\hline \multicolumn{14}{|l|}{ Menopausal State } \\
\hline Premenopausal & 10 & 0.007 & 0.01 & 0.297 & 0.087 & 0.71 & 0.971 & 0.063 & 0.087 & 0.81 & 0.73 & 0.118 & 0.301 \\
\hline Postmenopausal & 10 & & & & & & & & & & & & \\
\hline \multicolumn{14}{|l|}{ Parity } \\
\hline yes & 12 & 0.574 & 0.591 & 0.112 & 0.397 & 0.035 & 0.91 & 0.054 & 0.314 & 0.831 & 0.79 & 0.071 & 0.068 \\
\hline No & 8 & & & & & & & & & & & & \\
\hline \multicolumn{14}{|l|}{ ER status } \\
\hline Negative & 9 & 0.75 & 0.074 & 0.221 & 0.319 & 0.345 & 0.208 & 0.614 & 0.068 & 0.324 & 0.217 & 0.007 & 0.864 \\
\hline Positive & 11 & & & & & & & & & & & & \\
\hline \multicolumn{14}{|l|}{ PR status } \\
\hline Negative & 8 & 0.904 & 0.253 & 0.812 & 0.627 & 0.121 & 0.876 & 0.531 & 0.064 & 0.053 & 0.072 & 0.065 & 0.907 \\
\hline Positive & 12 & & & & & & & & & & & & \\
\hline \multicolumn{14}{|c|}{ Her2 overexpression } \\
\hline Negative & 13 & 0.523 & 0.739 & 0.513 & 0.201 & 0.257 & 0.384 & 0.201 & 0.087 & 0.987 & 0.201 & 0.214 & 0.259 \\
\hline Positive & 7 & & & & & & & & & & & & \\
\hline \multicolumn{14}{|c|}{ Lymph Node Involvement } \\
\hline No & 7 & 0.084 & 0.315 & 0.07 & 0.411 & 0.487 & 0.214 & 0.187 & 0.458 & 0.573 & 0.72 & 0.571 & 0.201 \\
\hline Yes & 13 & & & & & & & & & & & & \\
\hline \multicolumn{14}{|l|}{ Tumor Size } \\
\hline T1-T2 & 6 & 0.091 & 0.082 & 0.741 & 0.841 & 0.385 & 0.871 & 0.397 & 0.097 & 0.067 & 0.854 & 0.197 & 0.154 \\
\hline $\mathrm{T} 3-\mathrm{T} 4$ & 14 & & & & & & & & & & & & \\
\hline \multicolumn{14}{|l|}{ Tumor Grade } \\
\hline II & 7 & 0.372 & 0.218 & 0.397 & 0.871 & 0.222 & 0.287 & 0.179 & 0.214 & 0.91 & 0.418 & 0.227 & 0.128 \\
\hline III & 13 & & & & & & & & & & & & \\
\hline \multicolumn{14}{|l|}{ Metastasis } \\
\hline No & 16 & 0.09 & 0.55 & 0.433 & 0.287 & 0.454 & 0.321 & 0.074 & 0.27 & 0.852 & 0.082 & 0.812 & 0.875 \\
\hline Yes & 4 & & & & & & & & & & & & \\
\hline
\end{tabular}

Bold letters represent $\mathrm{p}<0.05$, significant

rho, $0.615 ; \mathrm{p}=0.04)$, as were miR-30b and $\mathrm{miR}-337-5 \mathrm{p}$ (Spearman's rho, 0.598; $\mathrm{p}=0.005$ ). Note that miR-342$3 \mathrm{p}$ expression did not correlate with other miRNAs except miR-342-5p (Spearman's rho, 0.724; p=0.001). Nonetheless, mir-29a expression profiles did not correlate with other miRNAs (Table 3).

In inflammatory breast cancer, miRNAs correlation profiles were different from non-inflammatory breast cancer. We demonstrated that less correlation was observed in miRNAs expression profiles in IBC compared to that in non-IBC. miR-486-3p expression levels correlate strongly with miR-342-5p (Spearman's rho $1.000 ; \mathrm{p}=0.000$ ) but not with mir-15a as were in non-IBC. Contrary to his correlation profiles in non-IBC, serum miR-451 expression in IBC did not correlate with other miRNAs. Astonishingly, miR-342-5p and miR-342-3p did not significantly correlate, although they belong to the same family and co-localized. There was a slight correlation between miR-320 and mir-15a as compared to that across non-IBC (Spearman's rho 0.590; $\mathrm{p}=0.016$ ) (Table 3).
A weak miRNAs correlation number was assessed in controls group. With the exception of miR-486-3p, miR-342-3p and miR-342-5p, there was no significant correlation showed in expression profiles of the miRNAs selected (Table 4).

Correlation between Circulating MicroRNA expressions and the clinic-pathologic features of patients with inflammatory and non- inflammatory Breast cancer.

Our first goal was to investigate the potential association of serum miRNA expression with tumor characteristics. We first measured the expression of 12 miRNAs, which have been selected based on previously supported roles in cancer progression and/or diagnostic utility. As shown in Table 5, in non-inflammatory breast cancer group, miR-24 and miR-335 level were correlated with Menopausal status $(\mathrm{p}=0.01$ and $\mathrm{p}=0.007$ respectively), the plasma levels of miR-335 were higherly expressed in Pre-menopausal patients than in Post-menopausal. However, Post-menopausal breast cancer patients had a significantly higher plasma levels of miR-24 than Pre-menopausal breast cancer patients. 
Table 6. Relationship between miRs Expression and Clinical and Histopathology Presentations in Inflammatory Breast Cancer Patients

\begin{tabular}{|c|c|c|c|c|c|c|c|c|c|c|c|c|c|}
\hline \multicolumn{14}{|c|}{ Inflammatory Breast Cancer p-value } \\
\hline $\begin{array}{c}\text { Patients } \\
\text { presentations }\end{array}$ & $\mathrm{N} 0$ & $\begin{array}{c}\text { miR- } \\
335\end{array}$ & $\begin{array}{c}\text { miR- } \\
24\end{array}$ & $\begin{array}{c}\text { miR- } \\
320\end{array}$ & $\begin{array}{l}\mathrm{miR}- \\
548 \mathrm{~d}- \\
5 \mathrm{p}\end{array}$ & $\begin{array}{l}\text { miR- } \\
342-5 p\end{array}$ & $\begin{array}{c}\mathrm{miR}- \\
15 \mathrm{a}\end{array}$ & $\begin{array}{c}\mathrm{miR}- \\
342- \\
3 \mathrm{p}\end{array}$ & $\begin{array}{c}\text { miR- } \\
29 a\end{array}$ & $\begin{array}{c}\mathrm{miR}- \\
451\end{array}$ & $\begin{array}{c}\text { miR- } \\
30 \mathrm{~b}\end{array}$ & $\begin{array}{c}\text { miR- } \\
337- \\
5 p\end{array}$ & $\begin{array}{c}\mathrm{miR}- \\
486- \\
3 \mathrm{p}\end{array}$ \\
\hline \multicolumn{14}{|l|}{ Age } \\
\hline$\geq 40$ years & 9 & 0.413 & 0.698 & 0.098 & 0.874 & 0.074 & 0.354 & 0.187 & 0.249 & 0.193 & 0.094 & 0.297 & 0.774 \\
\hline$<40$ years & 11 & & & & & & & & & & & & \\
\hline \multicolumn{14}{|l|}{ Menopausal State } \\
\hline Premenopausal & 10 & 0.224 & 0.154 & 0.347 & 0.056 & 0.634 & 0.157 & 0.012 & 0.192 & 0.259 & 0.454 & 0.576 & 0.312 \\
\hline Postmenopausal & 10 & & & & & & & & & & & & \\
\hline \multicolumn{14}{|l|}{ Parity } \\
\hline yes & 12 & 0.881 & 0.844 & 0.816 & 0.95 & 0.359 & 0.935 & 0.72 & 0.457 & 0.617 & 0.892 & 0.524 & 0.12 \\
\hline No & 8 & & & & & & & & & & & & \\
\hline \multicolumn{14}{|l|}{ ER status } \\
\hline Negative & 3 & 0.76 & 0.074 & 0.282 & 0.199 & 0.454 & 0.208 & 0.108 & 0.456 & 0.676 & 0.35 & 0.767 & 0.697 \\
\hline Positive & 17 & & & & & & & & & & & & \\
\hline \multicolumn{14}{|l|}{ PR status } \\
\hline Negative & 4 & 0.97 & 0.329 & 0.43 & 0.302 & 0.371 & 0.483 & 0.79 & 0.812 & 0.405 & 0.413 & 0.347 & 0.297 \\
\hline Positive & 16 & & & & & & & & & & & & \\
\hline \multicolumn{14}{|c|}{ Her2 overexpression } \\
\hline Negative & 13 & 0.989 & 0.178 & 0.138 & 0.394 & 0.304 & 0.001 & 0.058 & 0.053 & 0.074 & 0.421 & 0.39 & 0.297 \\
\hline Positive & 7 & & & & & & & & & & & & \\
\hline \multicolumn{14}{|c|}{ Lymph Node Involvement } \\
\hline No & 2 & 0.09 & 0.682 & 0.351 & 0.671 & 0.487 & 0.13 & 0.102 & 0.241 & 0.251 & 0.935 & 0.925 & 0.061 \\
\hline Yes & 18 & & & & & & & & & & & & \\
\hline \multicolumn{14}{|l|}{ Tumor Size } \\
\hline $\mathrm{T} 1-\mathrm{T} 2$ & 0 & 0.884 & 0.091 & 0.682 & 0.926 & 0.35 & 0.671 & 0.487 & 0.13 & 0.102 & 0.241 & 0.215 & 0.935 \\
\hline T3-T4 & 20 & & & & & & & & & & & & \\
\hline \multicolumn{14}{|l|}{ Tumor Grade } \\
\hline II & 9 & 0.273 & 0.49 & 0.532 & 0.153 & 0.344 & 0.859 & 0.83 & 0.721 & 0.659 & 0.799 & 0.678 & 0.289 \\
\hline III & 11 & & & & & & & & & & & & \\
\hline \multicolumn{14}{|l|}{ Metastasis } \\
\hline No & 14 & 0.819 & 0.028 & 0.005 & 0.921 & 0.68 & 0.151 & 0.479 & 0.712 & 0.211 & 0.187 & 0.405 & 0.736 \\
\hline Yes & 6 & & & & & & & & & & & & \\
\hline
\end{tabular}

Bold letters represent $\mathrm{p}<0.05$, significant

Similarly, miR-342-5p level were significantly associated with parity $(\mathrm{p}=0.035)$.We noticed that miR-342-5p had a higher expression level in patients with previous parity than those without (Table 5; Figure 1).

To determinate whether miRNAs were linked to the clinicopathological parameters in IBC, Spearman correlation test analysis demonstrated significant correlations between the expression of miR-15a and tumors bearing hormone HER-2 $(\mathrm{p}=0.001)$. Results showed that HER-2 tumors had a higher expression level of miR-15a than HER-2 positive tumors implicating that HER-2 signalisation may have a role in the regulation of miR-15a in inflammatory breast cancer. Lower plasma level of miR-342-3p was observed in Post-menopausal inflammatory breast cancer patients $(\mathrm{p}=0.012)$ indicating that the discriminatory potential of miR-342-3p seems to be better for Pre-menopausal patients (Table 6; Figure 1).

\section{Discussion}

Considering circulating miRNAs as disease marker has the following advantages: Related to the diseases closely, especially in tumors; Blood samples are available noninvasively; Subtle individual differences in the population; Stably present in serum/plasma; Mature methology of miRNA detection technology (Zeng et al., 2012). These advantages indicate that measuring circulating miRNA levels can be beneficial for early IBC and non-IBC 
B
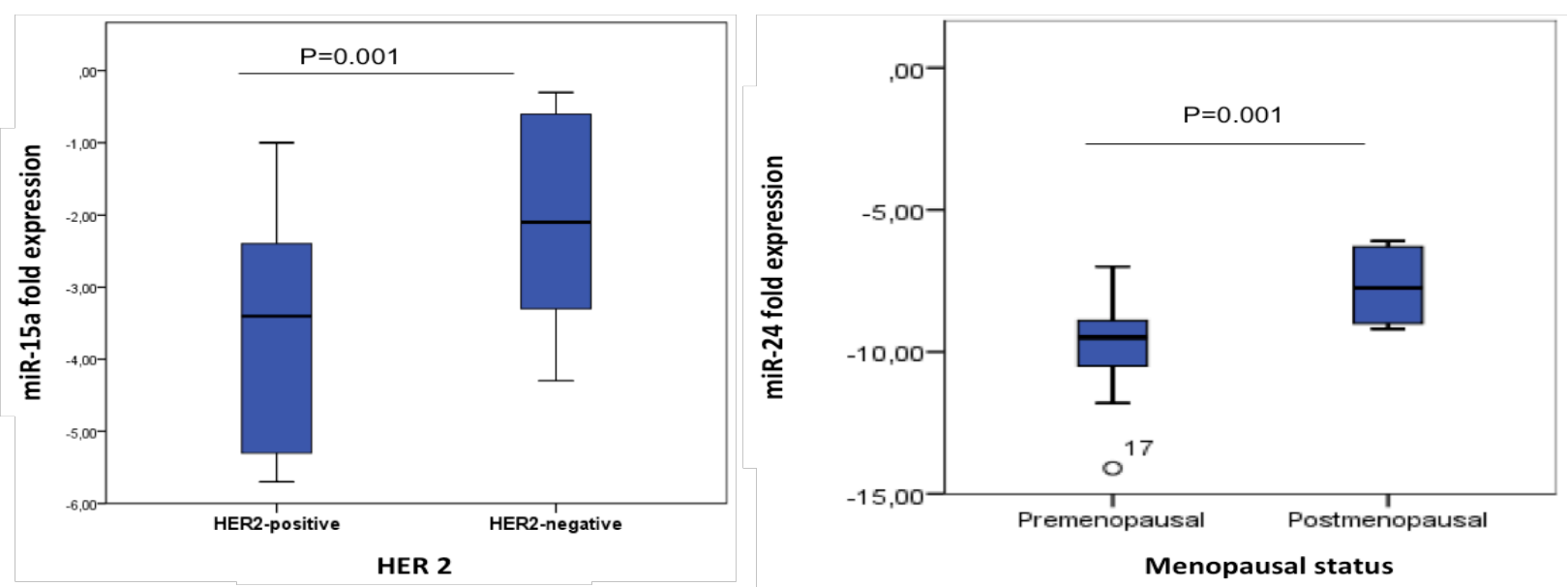

Figure 1. Serum miRNAs Levels in Patients with IBC and non-IBC. The box plots show: A) the fold change of the significantly differentially expressed miR-335 and miR-24 upon non-IBC groups based on menopausal status and miR-342-5p based on parity profile; B) the fold change of the significantly differentially expressed miR-15a and miR-24 upon IBC groups according to HER-2 and menopausal status respectively. The differences in serum levels were evaluated using the Mann-Whitney U test, and $\mathrm{p}$ values are indicated above the plots

screening and detection. Several major profiling platforms are used today in miRNAs detection. A powerful method for the analysis of serum miRNAs involves relative quantification by stem-loop RT-PCR. This method has been widely used for the sensitive detection of low abundance circulating miRNAs (Chen et al., 2005).

The importance of circulating miRNA in the prognostic value in breast carcinoma has been gained much attention. $\mathrm{Wu}$ and colleagues found that miR-21and miR-29 were significantly upregulated in the serum of breast cancer patients and may be useful biomarkers for breast cancer detection (Wu et al., 2011). Roth et al, who also analyzed serum samples, found that miR-155 can discriminate between non metastatic breast cancer patients and healthy individuals (Roth et al., 2010). Moreover, Anfossi et al, who investigated plasma samples from IBC patients, non-IBC and healthy women, found that high levels of serum miR-19a may represent a biomarker for IBC that is predictive for favorable clinical outcome in patients with metastatic HER2+ IBC (Anfossi et al., 2014).

In the current study, we analyzed the level of miRNAs to serve as an early detection marker of non-IBC and IBC by analyzing 12 circulating miRNAs (miR-342--3p, miR-320, miR-30b, miR-342-5p, miR-24, miR-15a, miR548d-5p, miR-486-3p, miR-29a, miR-451, miR-337-5p, miR-335) in serum of IBC patients $(\mathrm{N}=20)$, non-IBC patients $(\mathrm{N}=20)$ and healthy controls $(\mathrm{N}=20)$.

As the levels of circulating miRNAs may reflect the clinicopathologic status of cancer patients, the association between the miRNAs expression levels in the serum and the clinicopathological characteristics of the patients was investigated in the present study to assess the prognosis value of miRNAs in IBC and non-IBC. miR-24 expression in serum of postmenopausal non-IBC patients was significantly higher compared with the premenopausal non-IBC patients. While we are the first to demonstrate the prognostic potential of Circulating miR-24 in breast cancer, its prognostic potential was supported by a study that found high miR-24 expression in HCC cases with larger tumor size (Liu et al., 2014). Whereas, a tendency to higher levels of miR-335 was observed in the blood of premenopausal non-IBC patients indicating that the postmenopausal status may affect miR-335 expression levels. This observation is in the same line with other studies showing that menopausal status may deregulate level of miRNAs in breast cancer. In fact, miR-155 was upregulated in postmenopausal samples made it a considerable breast cancer biomarker for postmenopausal patients (Nassar et al., 2014). Thus, miR-24 may be a considerable non-IBC biomarker for postmenopausal patients and miR-335 for premenopausal non-IBC patients which could help the diagnosis of early onset breast cancer in Tunisian non-IBC patients. We also found that miR$342-5 p$ was upregulated in patients with positive parity as compared with those with nulliparity. Further, in the IBC samples, we found that serum miR-342-5p levels was downregulated in postmenopausal IBC samples indicating that the postmenopausal status may affect miR-342-5p expression levels.

Serum miR-15a levels were significantly lower in Her2+ IBC patients than in patients with HER2- IBC patients implying that miR-15a expression levels may be altered by HER2/neu overexpression in Her2+ IBC patients. Thus, it can be hypothesized that tumor cells overexpressed HER2 may contribute to the decreased miR-15a levels in the serum of Her2+ IBC patients. In this regard, it has been shown that HER $2 \Delta 16$ (a clinically important oncogenic isoform of HER2) can suppresses miR-15a (Cittelly et al., 2010). In addition, miR-15a was shown to mediate cell cycle arrest and promoting cell apoptosis of breast cancer by directly targeting Cyclin E1 suggesting the tumor suppressive activity of miR-15a in a breast cancer cell line (Luo et al., 2013; Shinden et al., 2015). Moreover, the identification of HER2/neu-induced miR-15a repression provides a greater understanding into how HER2/neu overexpression promotes invasion and 
metastasis in IBC.

miRNAs involvement in the pathogenesis of human cancers has been well documented. However, coexpression and co-regulation of miRNAs seems to be less pronounced. Through our analysis we observe a difference in miRNAs correlation profiles between IBC, non-IBC and healthy controls indicating important changes in molecular pathways in cancer cells. Many pairs of miRNAs were highly correlated with each other in both breast cancer groups, the largest pairwise miRNAs correlation was observed in non-IBC group suggesting that correlated miRNAs may be under similar transcriptional control, co-expressed together in exosomes.

Note that, correlation analysis is complementary to expression analysis, since two miRNAs may be highly correlated regardless of whether either one shows any mean change across groups (Lugli et al., 2015).

Interestingly, we found that miR-486-5p is still the most connected miRNA in IBC, non-IBC and healthy controls. In fact, miR-486-5p correlates perfectly with miR-342-3p in controls but not in breast cancer group in which miR-486-5p was correlated with miR-342-5p in IBC cases and miR-15a non-IBC group. This suggests changes in the regulatory network that occur in IBC and non-IBC. Moreover, it was described that miR-486-5p plays a tumor suppressor role in breast cancer by direct targeting PIM-1 oncogene (Zhang et al., 2014).

In addition, miR-30b and miR-337-5p interacts strongly together according to Spearman's rank correlation coefficient in non-IBC group. Moreover, In our previous studies it has been shown that high level of miR-337-5p and miR-30b may represent a new efficient biomarkers for early diagnosis for non-IBC patients. This co-up regulation provides additional evidence that miR-337-5p and miR$30 \mathrm{~b}$ may contribute together to promote initiation and development of non-IBC. This observation is in agreement with other studies showing that these miRNAs can promote the evolution of malignant behaviors by enhancing cellular invasion and immunosuppression (Gaziel-Sovran et al., 2011). Likewise, miR-320 was highly correlated with miR-24 and miR-548d-5p. A possible explanation is that these miRNAs may be secreted into the circulation by the same mechanisms. Nevertheless, the significant biological effect of correlated miRNAs on clinical pathology of breast cancer need to be further investigated.

In inflammatory breast cancer, miRNAs correlation profiles were different from non-inflammatory breast cancer. Thus, miR-320 revealed significant correlation with miR-15a expression. Interestingly, correlation analyses revealed no significant association of miR-451 with others miRNAs. A possible explanation for this finding is that exosomes containing miR-451, which is the most downregulated miRNAs in IBC, with an average decrease of 57.5-fold according to our previous IBC and non-IBC circulating miRNAs expression profile, are released into the circulation to a lesser extent in IBC subjects and processed independently of other miRNAs in IBC.

Astonishingly, miR-342-5p and miR-342-3p did not significantly correlate in IBC, although they belong to the same family. This might give reason to speculate that these two miRNAs may have the same transcription and maturation process, with different use and intake by IBC tumor cell, or different target. However, the appearance of significant strong correlation between miR-342-5p and miR-342-3p in both non-IBC and controls group may indicate important changes in molecular pathways in IBC versus non-IBC and controls.

Lastly, a weak miRNAs correlation number was observed in controls group as compared to IBC and nonIBC miRNAs correlation profile. Indeed, except miR-486$3 p$, miR-342-3p and miR-342-5p which were pairwise correlated as mentioned and discussed above, there was no significant correlation observed among other miRNAs. Thus, the loss of miRNAs correlation in healthy controls could represent an overall change in the regulatory network that occur in breast cancer cells during cancer progression, leading to the preferential co-regulation and co-expression of miRNAs in breast cancer group in opposition to healthy controls. These findings indicated that, as a novel noninvasive biomarker, circulating miRNAs would provide considerable diagnostic values in screening breast cancer.

Conclusion

This study is a continuity of our previous study in which we have shown that miR-451could be considered as a serological marker for IBC. However, miR-337-5p and miR-30b could discriminate non-IBC patients. Our current study showed that, in non-IBC group, the expression of circulating miR-335 and miR-24 was correlated with the menopausal state. Also, miR-342-5p was positively correlated with parity. We showed also that higher levels of miR-15a and miR-24 was positively correlated with Her2 status and menopausal state respectively in IBC patients. Moreover, we noticed that miRNAs interaction were different between IBC, non-IBC and healthy controls indicating important changes in molecular pathways in cancer cells and different mechanisms of carcinogenesis between the two Breast cancer subtype that may contribute to the dynamic of miRNAs profiles. Future large studies are warranted to validate our preliminary results and further allow us to examine whether miRNAs alterations are notably related to risk, onset or progression of the breast cancer and indicate their potential as molecular biomarkers for early cancer diagnosis, prognostic and facilitate the development of novel strategies for cancer therapy.

\section{Acknowledgements}

This work was supported in part by a travel grant from the American Association for the Advancement of Science (AAAS) (grant No S-LMA QM-09-GR-055 P.Is $\mathrm{JB}$ and $\mathrm{AB}$ ) and through development funds from the Lombardi Comprehensive Cancer Center. The authors would like to thank all the members of Laboratory of Genetic Immunology, Human Pathology (Tunis, Tunisia) and Departments of Oncology, Surgery and Microbiology, Georgetown University, Washington, DC, USA and the 
clinical staff for supporting the confirmation of cases in this study.

\section{References}

Anfossi S, Giordano A, Gao H, et al (2014). High serum miR19a levels are associated with inflammatory breast cancer and are predictive of favorable clinical outcome in patients with metastatic HER2+ inflammatory breast cancer. PLoS One, $9,83113$.

Chen C, Ridzon DA, Broomer AJ, et al (2005). Real-time quantification of microRNAs by stem-loop RT-PCR. Nucleic Acids Res, 33, e179.

Chen X, Ba Y, Ma L, et al (2008). Characterization of microRNAs in serum: a novel class of biomarkers for diagnosis of cancer and other diseases. Cell Res, 18, 997-1006.

Cittelly DM, Das PM, Salvo VA, et al (2010). Oncogenic HER2\{Delta\} 16 suppresses miR-15a/16 and deregulates BCL-2 to promote endocrine resistance of breast tumors. Carcinogenesis, 31, 2049-57.

Cortez MA, Bueso-Ramos C, Ferdin J, et al (2011). MicroRNAs in body fluids--the mix of hormones and biomarkers. Nat Rev Clin Oncol, 8, 467-77.

Croce CM (2009). Causes and consequences of microRNA dysregulation in cancer. Nat Rev Genet, 10, 704-14.

Dawood S, Cristofanilli M (2011). Inflammatory breast cancer: what progress have we made? Oncol, 25, 264-73.

Dawood S, Merajver SD, Viens P, et al (2011). International expert panel on inflammatory breast cancer: consensus statement for standardized diagnosis and treatment. Ann Oncol, 22, 515-23.

Fang Z, Tang J, Bai Y, et al (2015). Plasma levels of microRNA-24, microRNA-320a, and microRNA-423-5p are potential biomarkers for colorectal carcinoma. $J$ Exp Clin Cancer Res, 34, 86.

Gaziel-Sovran A, Segura MF, Di Micco R, et al (2011). miR$30 \mathrm{~b} / 30 \mathrm{~d}$ regulation of GalNAc transferases enhances invasion and immunosuppression during metastasis. Cancer Cell, 20, 104-18.

Hagrass HA, Sharaf S, Pasha HF, et al (2015). Circulating microRNAs - a new horizon in molecular diagnosis of breast cancer. Genes Cancer, 6, 281-7.

Hamdi K, Goerlitz D, Stambouli N, et al (2014). miRNAs in Sera of Tunisian patients discriminate between inflammatory breast cancer and non-inflammatory breast cancer. Springerplus, 3, 636.

Hatse S, Brouwers B, Dalmasso B, et al (2014). Circulating MicroRNAs as easy-to-measure aging biomarkers in older breast cancer patients: correlation with chronological age but not with fitness/frailty status. PLoS One, 9, 110644.

Hausler SF, Keller A, Chandran PA, et al (2010). Whole bloodderived miRNA profiles as potential new tools for ovarian cancer screening. Br J Cancer, 103, 693-700.

Heneghan HM, Miller N, Kelly R, et al (2010). Systemic miRNA-195 differentiates breast cancer from other malignancies and is a potential biomarker for detecting noninvasive and early stage disease. Oncologist, 15, 673-82.

Huang Z, Huang D, Ni S, et al (2010). Plasma microRNAs are promising novel biomarkers for early detection of colorectal cancer. Int J Cancer, 127, 118-26.

Kodahl AR, Lyng MB, Binder H, et al (2014). Novel circulating microRNA signature as a potential non-invasive multimarker test in ER-positive early-stage breast cancer: a case control study. Mol Oncol, 8, 874-83.

Konishi H, Ichikawa D, Komatsu S, et al (2012). Detection of gastric cancer-associated microRNAs on microRNA microarray comparing pre-and post-operative plasma. $\mathrm{Br} J$
Cancer, 106, 740-7.

Li X, Abdel-Mageed AB, Mondal D, et al (2013). MicroRNA expression profiles in differentiated thyroid cancer, a review. Int J Clin Exp Med, 6, 74-80.

Liu YX, Long XD, Xi ZF, et al (2014). MicroRNA-24 modulates aflatoxin B1-related hepatocellular carcinoma prognosis and tumorigenesis. Biomed Res Int, 2014, 482926.

Livak KJ, Schmittgen TD (2001). Analysis of relative gene expression data using real-time quantitative PCR and the 2(-Delta Delta C(T)) Method. Methods, 25, 402-8.

Lodes MJ, Caraballo M, Suciu D, et al (2009). Detection of cancer with serum miRNAs on an oligonucleotide microarray. PLoS One, 4, 6229.

Lugli G, Cohen AM, Bennett DA, et al (2015). Plasma exosomal miRNAs in persons with and without alzheimer disease: altered expression and prospects for biomarkers. PLoS One, 10, 139233.

Luo Q, Li X, Li J, et al (2013). MiR-15a is underexpressed and inhibits the cell cycle by targeting CCNE1 in breast cancer. Int J Oncol, 43, 1212-8.

Mitchell PS, Parkin RK, Kroh EM, et al (2008). Circulating microRNAs as stable blood-based markers for cancer detection. Proc Natl Acad Sci USA, 105, 10513-8.

Nassar FJ, El Sabban M, Zgheib NK, et al (2014). miRNA as potential biomarkers of breast cancer in the Lebanese population and in young women: a pilot study. PLoS One, 9, 107566.

Palmero EI, de Campos SG, Campos M, et al (2011). Mechanisms and role of microRNA deregulation in cancer onset and progression. Genet Mol Biol, 34, 363-70.

Roth C, Rack B, Muller V, et al (2010). Circulating microRNAs as blood-based markers for patients with primary and metastatic breast cancer. Breast Cancer Res, 12, 90.

Sayed D, Abdellatif M (2011). MicroRNAs in development and disease. Physiol Rev, 91, 827-87.

Schwarzenbach H, Hoon DS, Pantel K (2011). Cell-free nucleic acids as biomarkers in cancer patients. Nat Rev Cancer, 11, 426-37.

Schwarzenbach H, Nishida N, Calin GA, et al (2014). Clinical relevance of circulating cell-free microRNAs in cancer. Nat Rev Clin Oncol, 11, 145-56.

Shapira I, Oswald M, Lovecchio J, et al (2014). Circulating biomarkers for detection of ovarian cancer and predicting cancer outcomes. Br J Cancer, 110, 976-83.

Shinden Y, Akiyoshi S, Ueo H, et al (2015). Diminished expression of MiR-15a is an independent prognostic marker for breast cancer cases. Anticancer Res, 35, 123-7.

Simpson RJ, Lim JW, Moritz RL, et al (2009). Exosomes: proteomic insights and diagnostic potential. Expert Rev Proteomics, 6, 267-83.

Tomimaru Y, Eguchi H, Nagano H, et al (2012). Circulating microRNA-21 as a novel biomarker for hepatocellular carcinoma. J Hepatol, 56, 167-75.

Van der Auwera I, Limame R, van Dam P, et al (2010). Integrated miRNA and mRNA expression profiling of the inflammatory breast cancer subtype. Br J Cancer, 103, 532-41.

Wang F, Zheng Z, Guo J, et al (2010). Correlation and quantitation of microRNA aberrant expression in tissues and sera from patients with breast tumor. Gynecol Oncol, 119, 586-93.

Wu C, Wang C, Guan X, et al (2014). Diagnostic and prognostic implications of a serum miRNA panel in oesophageal squamous cell carcinoma. PLoS One, 9, 92292.

Wu Q, Lu Z, Li H, et al (2011). Next-generation sequencing of microRNAs for breast cancer detection. J Biomed Biotechnol, 2011, 597145.

Wu Q, Wang C, Lu Z, et al (2012). Analysis of serum genome- 
$K$ Hamdi et al

wide microRNAs for breast cancer detection. Clin Chim Acta, 413, 1058-65.

Yamauchi H, Woodward WA, Valero V, et al (2012). Inflammatory breast cancer: what we know and what we need to learn. Oncologist, 17, 891-9.

Yan LX, Huang XF, Shao Q, et al (2008). MicroRNA miR-21 overexpression in human breast cancer is associated with advanced clinical stage, lymph node metastasis and patient poor prognosis. $R N A, \mathbf{1 4}, 2348-60$.

Zeng X, Xiang J, Wu M, et al (2012). Circulating miR-17, miR-20a, miR-29c, and miR-223 combined as non-invasive biomarkers in nasopharyngeal carcinoma. PLoS One, 7, 46367.

Zhang G, Liu Z, Cui G, et al (2014). MicroRNA-486-5p targeting PIM-1 suppresses cell proliferation in breast cancer cells. Tumour Biol, 35, 11137-45.

Zhu W, Qin W, Atasoy U, et al (2009). Circulating microRNAs in breast cancer and healthy subjects. BMC Res Notes, $2,89$. 